\title{
ANTECEDENTES ARQUEOLOGICOS DEL GENERO DIPLODON (SPIX, 1827) (BIVALVIA, HYRIIDAE) EN CHILE
}

\section{ARCHAEOLOGICAL RECORD FOR DIPLODON (SPIX, 1827) (BIVALVIA, HYRIIDAE) INCHILE}

\author{
Donald Jackson ${ }^{1} \&$ Douglas Jackson ${ }^{2}$ \\ ${ }^{1}$ Departamento de Antropología, Facultad de Ciencias Sociales, Universidad de Chile. \\ Casilla 10115, Santiago, Chile. Email: djackson@uchile.cl \\ ${ }^{2}$ Pedro Torres 830 Ñuñoa, Santiago.
}

\begin{abstract}
RESUMEN
Se presenta una revisión bibliográfica de los registros del género Diplodon (Spix, 1827) (Hyriidae), presentes en sitios arqueológicos de Chile. Las evidencias indican una efímera presencia de estos bivalvos en contextos arqueológicos de finales del Pleistoceno, mientras que durante gran parte del Holoceno su registro es más significativo. No obstante, sólo algunos sitios arqueológicos muestran su utilización como materia prima y/o recurso alimenticio. Las evidencias arqueológicas de Diplodon muestran similar distribución a la actual. Se discuten algunas implicancias paleoambientales y biogeográficas en relación a su distribución, así como la necesidad de vincular esta información con distintas disciplinas para entender la importancia de su rol en contextos arqueológicos.

Palabras claves: Diplodon, Hyriidae, Almeja de agua dulce, Arqueología, cronología, Chile.
\end{abstract}

\section{ABSTRACT}

We review bibliography on genus Diplodon (Spix, 1827) (Hyriidae) records obtained in archaeological contexts in Chile. Evidence indicates an ephemeral late Pleistocene presence of these bivalves, while its record increases significantly during the Holocene. Nevertheless, only few archaeological sites show its use as raw material and/or food resource. Diplodon archaeological evidence is similar to current genus distribution. We discuss some palaeoenvironmental and biogeographical implications of their distribution, as well as the need to relate this information with different disciplines in order to understand its importance in archaeological contexts.

KeYwords: Diplodon, Hyriidae, Freshwater mussel, archaeology, chronology, Chile.

\section{INTRODUCCION}

Durante el desarrollo de un trabajo de recopilación bibliográfica emprendido por los autores, con el objetivo de conocer los registros de Diplodon en contextos arqueológicos, se pudo constatar que estos bivalvos están escasamente documentados, no obstante que fueron consumidos por los primeros pobladores que llegaron a nuestro territorio.
Este bivalvo es el único molusco dulceacuícola que ha sido consumido por poblaciones prehistóricas en Chile y Argentina, incluso consumiéndose hasta nuestros días por mapuches y poblaciones locales del sur de Chile.

Las almejas de agua dulce (Diplodon), son bivalvos dulceacuícolas de la familia Hyriidae, que habitan sistemas hidrográficos continentales del centro-sur de Chile y Argentina. En Chile, se 
distribuye desde el río Mataquito, Curicó (345ㅇ's; $71^{\circ} 48^{\prime} \mathrm{W}$ ) hasta el río Negro en el istmo de Ofqui (463'ㅇ' $74^{\circ} 10^{\prime} \mathrm{W}$ ) (Parada \& Peredo 2006), no obstante recientes registros muestran su presencia en el río Grande,afluente del río Limarí

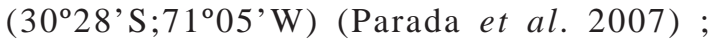
encontrándose en sustratos arenosos, pedregosos y fangosos (Lara \& Parada 2008). Son organismos filtradores, que se alimentan principalmente de fitoplancton, pero también de bacterias (Sepúlveda 1988), condición que podría ser utilizada como biofiltro (Lara et al. 2002).

Son bivalvos de forma oblonga-alargadas, que pueden alcanzar longitudes hasta los $85 \mathrm{~mm}$ (Lara et al. 1988), y sus valvas presentan un periostraco delgado de color pardo brillante, internamente nacarada con cavidad profunda en la impresión del músculo retractor anterior.

En cuanto a su abundancia, las almejas de agua dulce son frecuentes de encontrar, pero en pequeñas densidades, no existiendo antecedentes de grandes bancos, aunque su alta biomasa ha permitido proponerlos como un recurso alimenticio potencial. (Lara et al. 1988).

Las evidencias arqueológicas para este bivalvo son relativamente amplias pero dispersas en la literatura (Lara et al. 1988, Adán et al. 2004, Jackson 2007). En la mayoría de los casos, los especímenes encontrados han sido identificados sólo a nivel genérico, salvo algunas excepciones (Lara et al. 1988, Jackson 2007, Quiroz \& Martínez 2007). En Argentina también se tienen algunos registros arqueológicos (Prates \& Marsans 2007), y al igual que en Chile, su escasa presencia se debería principalmente a factores tafonómicos de conservación diferencial, especialmente por el alto grado de fragmentación de las valvas.

La identificación de especies es compleja y difícil a partir de restos de valvas fragmentadas, lo que, sumado a una fuerte variación fenotípica del género (Parada \& Peredo 2002, 2006), obliga a trabajar en conjunto con taxónomos, al menos en alguna interfase del trabajo interdisciplinario.

\section{METODOS}

Para comprender el estado actual de los registros de Diplodon en sitios arqueológicos, se realizó una revisión de las revistas especializas en arqueología y antropología, considerando también artículos publicados en revistas de ciencias naturales.

Se previlegió aquellos artículos donde la presencia de Diplodon contaba con registros estratigráficos o con dataciones radiocarbónicas, que permitieran su asociación con algún período cultural, de manera de obtener una perspectiva temporal lo más amplia posible, como también que incluyera gran parte del área de distribución, donde habitó esta especie. En general no se mencionan en el texto denominaciones específicas, salvo que ellas estén bien fundamentadas, considerándose en el texto sólo como «almejas de agua dulce», o bajo el nombre genérico de Diplodon sp.

\section{RESULTADOS}

Los antecedentes bibliográficos consultados, proporcionaron información sobre la presencia y utilización de la «almeja de agua dulce», en diferentes sitios arqueológicos de Chile, durante el período comprendido desde el Pleistoceno final hasta el Holoceno tardío (Fig. 1).

Los registros más antiguos del posible aprovechamiento de Diplodon se han encontrado en el sitio arqueológico de Monte Verde ( $39^{\circ} \mathrm{S}$ ), situado a orillas del río Chinchi-Huapi, afluente del río Maullín en el sur de Chile (Dillehay 1984, 1997). Este sitio corresponde a un campamento de cazadores del final del Pleistoceno, datado en 12.500 años Antes del Presente (A.P.). Las evidencias están constituidas por precarios fragmentos de valvas y su escasa frecuencia sugiere un limitado consumo o bien que su desconche se desarrolló próximo a los lugares de recolección, trasladando únicamente las partes comestibles al campamento, sin embargo no debe excluirse la re-depositación de ejemplares por subidas del río Chinchi-Huapi, durante la estación invernal.

En el sitio Paleoindio de Tagua Tagua ( $34^{\circ} \mathrm{S}$ ), de Chile Central, las evidencias arqueológicas asociadas a fauna extinta, datadas entre los 11.400 y 11.000 años A.P. (Tagua Tagua 1) y 10.200 y 9.700 años A.P. (Tagua Tagua 2) (Montané 1968, Núñez et al. 1994), no registran evidencias de Diplodon, no obstante que se trata de asentamientos emplazados en paleo-playas de un sistema lacustre, donde aún existe la presencia de este bivalvo. 
Gayana 72(2), 2008

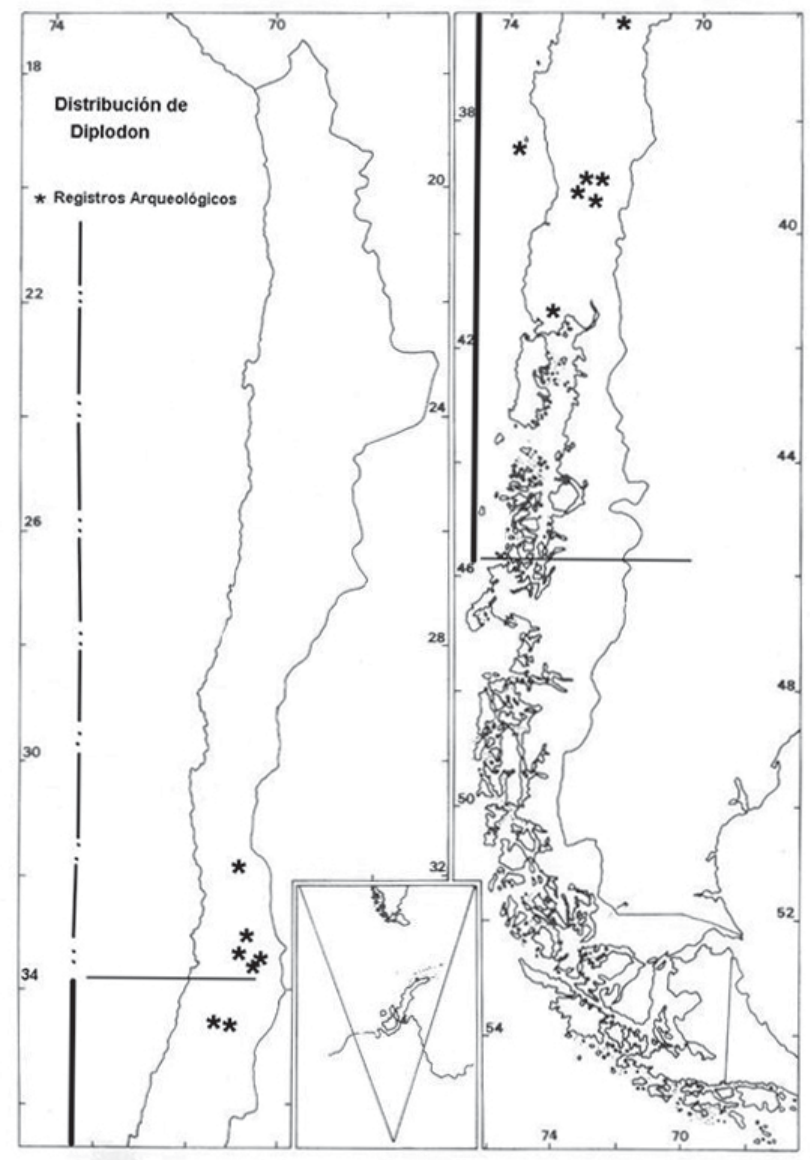

Figura 1: Distribución geográfica de Diplodon sp. (Hyriidae). Línea continua muestra la distribución actual y línea discontinua registros dudosos. Registros arqueológicos con asterisco.

Figure 1: Geographic Distribution of Diplodon sp. (Hyriidae). Solid line shows the current distribution and dubious records on dotted line. Archaeological records with asterisks.

Otro relevante asentamiento, se encuentra localizado también en la exlaguna de Tagua Tagua, en Cuchipuy $\left(34^{\circ} \mathrm{S}\right)$, un sitio habitacional emplazado en la ribera de la antigua laguna, sitio que presenta una larga y continua secuencia ocupacional. El estudio del contexto cultural y estratigráfico ha permitido discriminar cuatro niveles ocupacionales, tres correspondientes a grupos cazadores-recolectores del Holoceno Temprano y Medio con dataciones que se extienden cronológicamente desde el $8.070 \pm 100$ años A.P. al 5.060 \pm 170 años A.P. y un nivel correspondiente a grupos Alfareros (Kaltwasser et al. 1980, 1983). En estos tres últimos niveles ocupacionales se registran valvas de Diplodon, especialmente en el nivel 3 asociadas a otros restos de fauna acuática (anfibios, peces y aves), evidencias que atestiguan el aprovechamiento de los recursos del sistema lacustre. En el nivel ocupacional 2, donde aparecen numerosos entierros humanos, varios de ellos presentaban valvas de Diplodon dispuestas intencionalmente sobre la cara de los individuos a modo de ofrendas funerarias (Kaltwasser et al. 1986) 
Así mismo en el nivel 2 del sitio de Tagua Tagua 1, un pequeño campamento de cazadores-recolectores del Holoceno Medio, datado en 6.130£115 años A.P. (Montané 1969, Duran 1980), se registraron algunos fragmentos de Diplodon, asociados a otras especies de moluscos dulceacuícolas (Covacevich 1971). Estas evidencias son contemporáneas con los niveles intermedios del Holoceno Medio del sitio de Cuchipuy, datado en 6.105 \pm 145 años A.P. y $6.160 \pm 100$ años A.P. (Kaltwasser et al. 1983), confirmando así un sincronismo entre los eventos ocupacionales de ambas sitios.

En la X Región de Los Lagos, se ha excavado el sitio denominado Marifilo 1 ( $39^{\circ} \mathrm{S}$ ), que corresponde a un alero rocoso emplazado a unos 1260 metros de la ribera del lago Calafquén (Velásquez \& Adán 2002). Este sitio presenta una secuencia con varios eventos ocupacionales casi ininterrumpida, de lo que constituye un verdadero conchal de valvas de Diplodon, asociadas con algunos ejemplares de Chilina y restos culturales, correspondiente a grupos cazadores y recolectores del Holoceno Temprano (8.420 \pm 40 años A.P.) y un componente

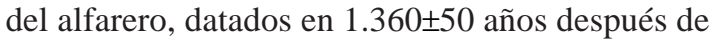
Cristo (d.C.) (Adán et al., 2004). Las evidencias de este sitio indican que se trataría de un campamento con ocupaciones reiteradas, donde este molusco dulceacuícola, constituyó un recurso relevante en la subsistencia de estos grupos.

En la IX Región se encuentra el sitio de Quillén I $\left(39^{\circ} \mathrm{S}\right)$, alero rocoso que presenta tres ocupaciones, las dos primeras correspondiente a cazadores y recolectores, datadas en $4.675 \pm 105$ años A.P. y $2.030 \pm 70$ años A.P. respectivamente y una tercera ocupación asignable a grupos del período Alfarero (sin datación); en este sitio se encontraron un total de 97 valvas de Diplodon (Valdés et al. 1982). Otras evidencias se han encontrado en la cueva de Quilmo, en Chillán, que presenta una ocupación acerámica y otra más tardía con alfarería, en un contexto similar al de Quillén I ( $\left.39^{\circ} \mathrm{S}\right)$, donde se presentan restos de Diplodon chilensis asociado a Chilina sp., y otros restos de fauna dulceacuícola y terrestre (Quiroz \& Martínez 1997). Evidencias similares se han constatado en el sitio Pucón VI (39 S) (Adán et al. 2004) y en la cueva de los Catalanes en la misma región (Berdichewsky 1968), así como también en sitios del período Alfarero de Isla Mocha (Gálvez 1997).

En alero Quino 1, situado a pocos kilómetros del sitio de Quillén I, se registró una ocupación del período alfarero, con evidencias culturales asociadas a Diplodon (Sánchez \& Inostroza 1985). La secuencia estratigráfica, desde el piso del asentamiento hasta niveles superiores, muestra un progresivo consumo de este molusco, cuyas tallas modales están entre 31 y 35 mm, parámetros métricos significativamente menores a los registrados actualmente en el mismo lugar (15 a 75 $\mathrm{mm}$ ), lo que ha permitido sugerir una sobreexplotación de este recurso en tiempos Prehispánicos (Lara et al. 1988).

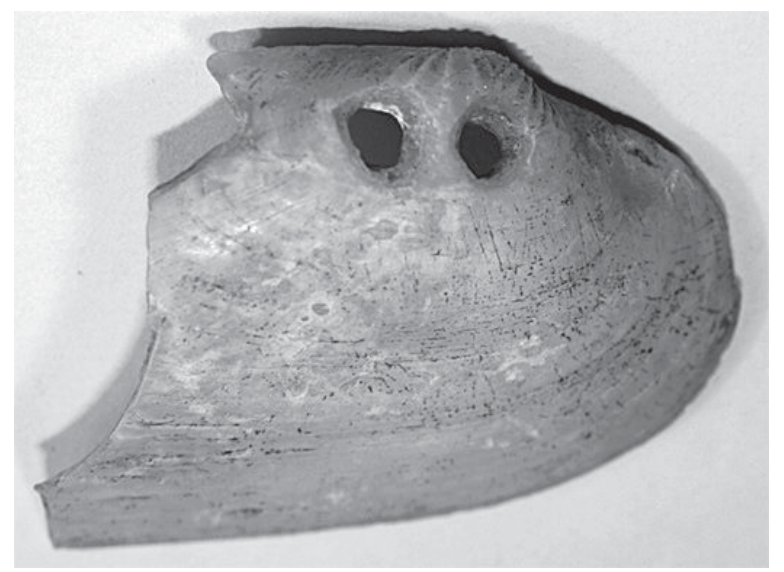

Figura 2: Ejemplar de Diplodon chilensis procedente del sitio arqueológico Blanca Gutiérrez (Chile central) con orificios culturales en el área sub-umbal, para ser usado como pendiente.

FIGURE 2: Specimen of Diplodon chilensis from archeological place of Blanca Gutiérrez (Chile central) with cultural holes on the sub-umbal area to be used as hanging. 
En los valles de Chile central, existen varios sitios del período Alfareros con presencia de Diplodon. El sitio, alero El Salitral, ubicado en el cordón cordillerano de Chacabuco ( $\left.32^{\circ} \mathrm{S}\right)$, con una ocupación correspondiente al alfarero, datado en $100 \pm 110$ años d.C., se registran tres fragmentos, uno de ellos perforado para ser utilizado como ornamento (Stehberg \& Pinto 1980).

Otros hallazgos en ocupaciones del período Alfarero Medio y Tardío se han constatado en varios sitios del Complejo Cultural Aconcagua, como el Coligüe (32 ${ }^{\circ} \mathrm{S}$ ), en el cordón de Chacabuco, datado en $990 \pm 100$ años d.C. y 1.050 130 años d.C. (Durán et al. 1993), y el sitio RML015 (Familia Fernández) (33 S), emplazado en las cercanías del estero de Lampa (Santiago) (Gaete 1993) o en el extenso sitio habitacional conocido como Blanca Gutiérrez

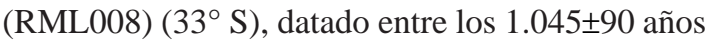
d.C. y los $1.420 \pm 60$ años d.C. (Pavlovic et al. 1998), donde el $29 \%$ de los moluscos, corresponden a Diplodon sp., que en algunos casos fueron utilizados como ornamentos (Fig.2) (Jackson 2007), al igual que en el sitio El Salitral (Stehberg \& Pinto 1980).

En el valle de Aconcagua, algo más al norte y próximo a la localidad de Catemu, se encuentra el sitio arqueológico Cerro La Cruz ( $32^{\circ} \mathrm{S}$ ), un enclave económico administrativo Incaico, datado entre los $1.285 \pm 70$ años d.C. y los $1.430 \pm 60$ años d.C., donde las excavaciones arqueológicas atestiguan una efímera presencia de este bivalvo (Rodríguez et al. 1993).

\section{DISCUSION Y CONCLUSIONES}

Un primer aspecto a considerar es la integridad de las valvas de Diplodon, que se encuentran en los contextos arqueológicos, las cuales están muy fragmentadas, dificultando la labor de identificación. Esto se debe probablemente a un debilitamiento del periostraco de las valvas, producto de factores tafonómicos como la erosión y constitución químicas de los substratos de los depósitos arqueológicos, como también del resultado de los procesos postdepositacionales que han afectado los sitios arqueológicos.

La fragmentación de las valvas, también se produce por factores antrópicos, como el pisoteo durante la ocupación de los asentamientos y por la técnica usada en la abertura de las valvas. Por último debe considerarse que los procedimientos de recuperación, embalaje, y transporte también actuarían sesgando la integridad de las valvas.

La densidad de valvas fracturadas, sólo puede ser controlada en la fase de trabajo arqueológico, mejorando las técnicas de recuperación y conservación de éstas. Este aspecto metodológico tiene gran relevancia, porque el grado de integridad de las valvas se encuentra en directa relación con sus posibilidades de estudio analítico, al poder controlar las tallas de las valvas y el número mínimo de individuos, permitiendo así calcular biomasa y estimar densidad poblacional de los sitios, como también permitirían una identificación más certera al conservarse los rasgos diagnósticos de los especímenes recuperados.

Como resultado de las condiciones del estado de conservación de las valvas, no siempre se ha logrado una identificación a nivel específico o ésta ha sido preliminar como en el caso de $D$. chilensis (Quiroz \& Martínez 2007). En concreto, sólo en escasos sitios arqueológico se ha identificado la especie (Lara et al. 1988), aunque existen otras identificaciones como Diplodon atratus (Stehberg \& Pinto 1980), que no son válidas o no están vigentes (Parada \& Peredo 2006).

Para la especie Diplodon solidulus, aún no se registran ejemplares en sitios arqueológicos, a pesar de las diversas excavaciones llevadas a cabo en las áreas de su distribución actual, al sur de la latitud $40^{\circ}$ (Parada \& Peredo 2006). Su ausencia puede deberse a una baja densidad poblacional o a la baja predectibilidad como recurso alimenticio.

En cuanto a la secuencia temporal de Diplodon, éste se encuentra desde el final del pleistoceno (12.500 años A.P.) en el sitio de Monte Verde (Dillehay 1997) hasta el Holoceno tardío, en sitios de Chile central. Durante toda esta secuencia fueron utilizados principalmente como una fuente de alimentación alternativa, como materia prima para fabricar adornos y también como ofrendas funerarias (Stehberg \& Pinto 1980, Jackson 2007, Kaltwasser et al. 1980).

Los resultados muestran que no existen grandes cambios en la frecuencia y tamaño de las valvas de algunos sitios, así como en Marifilo 1, donde la secuencia de este bivalvo es continua desde los $8.420 \pm 40$ años A.P. hasta los $1.360 \pm 50$ años A.P., donde no se observan cambios significativos en el tamaño de las valvas (Adán et al.2004). En cambio 
en otros sitios como Pucón VI (Adán et al. 2004) y Quillen 1 (Lara et al. 1988), la secuencia atestigua un aumento de las tallas de Diplodon desde los niveles inferiores más antiguos, a los niveles superiores más recientes, lo que podría deberse a opciones culturales más que a factores de disponibilidad por cambios paleoambientales, pues los antecedentes ecológicos indican una alta tolerancia del Diplodon chilensis a los cambios de temperatura (Lara et al. 1988).

Las evidencias palinológicas de la región indican que durante los 9.500 a los 3.000 años A.P., existieron especies vegetales más termófilas y resistentes a la sequía, y que, durante los 7000 años A.P., se registra el momento más cálido y seco en la secuencia (Villagrán et al.1995), lo que probablemente no afectó a las poblaciones de Diplodon que vivieron en la interfase Pleistoceno-Holoceno, debido a la tolerancia de esta especie a los cambios ambientales (Lara et al. 1988).

La frecuencia de valvas de Diplodon en la gran mayoría de los sitios sugiere que su consumo fue mínimo y ocasional, posiblemente fue un recurso obtenido en forma oportunista, que no constituyó un aporte importante en la dieta. Es probable que una sobreoferta de recursos bióticos en los ambientes lénticos, como por ejemplo en la antigua laguna de Tagua Tagua, excluyera el aprovechamiento más intensivo de aquellos recursos de menor biomasa como la Almeja de Agua Dulce.

No obstante lo anterior, algunos sitios sí representan un consumo intensivo como es el caso de Marifilo 1 , donde probablemente el género Diplodon representó un recurso de subsistencia tipo "snack". Este tipo de aprovechamiento probablemente no representó una sobre explotación, como se ha sugerido para otros sitios, sobre la base únicamente de la presencia de ejemplares muy pequeños (Lara et al. 1988).

Por otra parte, en algunos sitios como Cuchipuy, este bivalvo además de ser consumido fue objeto de ofrendas, disponiéndose valvas sobre los entierros humanos (Kaltwasser et al. 1987). En otros contextos fueron usadas como pendientes, debido probablemente al nacarado intenso de sus valvas, como los hallados en el alero El Salitral (Stehberg \& Pinto 1980) y en el sitio Blanca Gutiérrez (Jackson 2007).

En consecuencia, sólo se conoce la paleodistribución de Diplodon a nivel de género y algunos datos aislados para Diplodon chilensis en el sur de Chile. No existe información de contextos arqueológicos con presencia de Diplodon solidulus ni tampoco de las sub-especies de $D$. chilensis. La paleo-distribución del género Diplodon, se extendería al sur hasta los $40^{\circ} \mathrm{S}$ en el área del lago Calafquén y por el norte a los $31^{\circ} \mathrm{S}$ en el curso superior del río Illapel. Esto es aproximadamente coincidente con la distribución actual de Diplodon chilensis situado entre los $35^{\circ}$ y $45^{\circ} \mathrm{S}$ (Parada \& Peredo 2006), aunque recientes registros muestran su presencia al norte hasta los $30^{\circ} \mathrm{S}$ (Parada et. al. 2007). La eventual ausencia de registros arqueológicos más al norte y sur, se debe probablemente a un sesgo en los muestreos arqueológicos.

Debemos resaltar que las evidencias arqueológicas de Diplodon constituyen un material de estudio no explorado para el conocimiento de su rol en las sociedades prehispánicas, así como también para abordar diversos temas biológicos en relación a problemas de taxonomía, paleo-distribución y biogeográficos. Una perspectiva de estudios sistemáticos interdisciplinarios parece ser prometedora en la investigación de estas problemáticas.

\section{AGRADECIMIENTOS}

Nuestros agradecimientos a Eileen Riedemann por el trabajo fotográfico, Leonor Adán por proporcionar información inédita del sitio de Marifilo 1, Andrés Troncoso por la revisión del manuscrito y a César Méndez por la confección del Abstract.

\section{BIBLIOGRAFIA}

Adán, L., E. Mera, M. Becerra \& M. Godoy. 2004. Ocupación Arcaica en el territorio boscoso y lacustre de la Región Precordillerana Andina del Centro-Sur de Chile. El sitio Marifilo 1 de la localidad de Pucura. Chungará. Volumen Especial, Tomo II: 1121-1136.

Berdichewsky, B.1968. Excavaciones en la cueva de los Catalanes. Boletín de Prehistoria de Chile, 1: 33-38.

Covacevich, V. 1971. Los moluscos Pleistocénicos y Holocénicos de San Vicente de Tagua Tagua. Memoria para optar al título de Geólogo, Departamento de Geología, Facultad de Ciencias Físicas y Matemáticas, Universidad de Chile. 82 p. 
DillehAY, T. 1984. A Late ice-age settlement in southern Chile. Scientific American 251 (4):100-109.

Dillehay, T. 1997. Monte Verde: A Late Pleistocene Settlement in Chile. Vol.2; The Archaeological Context. Smithsonian Institution Press, Washington D.C.

Durán, E. 1980. Tagua Tagua II, Nivel de 6.130 años. Descripción y relaciones. Boletín del Museo Nacional de Historia Natural (Chile), 37:75-86.

Durán, E., A. Rodríguez \& C. González. 1993. Sistemas adaptativos de Prehispánicas en el cordón de Chacabuco. Boletín Museo Regional de La Araucanía 4: 235-248.

Gaete, N. 1993. R.M.L. 015 “Familia Fernández”; Análisis de un contexto Aconcagua atípico en Chile Central. Boletín Museo Regional de La Araucanía 4: 249-262.

GÁLVEz, O. 1997. Análisis de restos malaco-arqueológicos de la Isla Mocha. En “La Isla de las palabras rotas” (Quiroz D. y R. Sánchez Comp.), Pp. 195-213, Colección de Antropología IV, Centro de Investigaciones Diego Barros Arana, Dirección de Bibliotecas, Archivos y Museos.

Kaltwasser, J., A. Medina \& J. Munizaga. 1980. Cementerio del Periodo Arcaico en Cuchipuy. Revista Chilena de Antropología 3:109-123.

Kaltwasser, J., A. Medina \& J. Munizaga. 1983. Estudio de once fechas de R.C. 14 relacionadas con el Hombre de Cuchipuy. Boletín de Prehistoria de Chile 9: 9-13.

Kaltwasser, J., A. Medina, E. Aspillaga \& C. Paredes. 1986. El hombre de Cuchipuy; Prehistoria de Chile Central en el período Arcaico. Chungará 16-17: 99-105.

JACKSON, D. 2007. Identificación e interpretación de moluscos asociados al sitio habitacional Blanca Gutiérrez (RML-008) del Complejo Cultural Aconcagua, Chile Central. (Manuscrito no publicado).

Lara, G., E. Parada, S. Peredo, J. Inostroza \& H. Mora. 1988. La almeja de agua dulce Diplodon chilensis (Gray, 1828), Un recurso potencial. Boletín Museo Regional de La Araucanía, Temuco, 3:3340.

LARA, G. \& E.PARADA. 2008. Mantención del patrón de distribución espacial, densidad y estructura de tamaños de la almeja de agua dulce Diplodon chilensis Gray,1828 (Bivalvia: Hiriidae) en el Lago Panguipulli, Chile. Gayana 72 (1): 45-51.

Montané, J. 1968. Paleo-Indio remains from Laguna Taguatagua, Central Chile. Science 161: $1137-$ 1138.

Montané, J. 1969. Fechado del nivel superior de Tagua Tagua. Noticiario Mensual del Museo Nacional de Historia Natural Año XIV, N 161:9-10.

NúÑEz, L., J. Varela, R. CASAmiquela, V. Schiappacasse, H. Niemayer \& C. Villagran. 1994. Cuenca de Taguatagua en Chile: el ambiente del Pleistoceno Superior y ocupaciones humanas. Revista Chilena de Historia Natural 67:503-519.
Parada, E. \& S. Peredo. 2002. Estado actual de la taxonomía de bivalvos dulceacuícolas chilenos: progresos y conflictos. Revista Chilena de Historia Natural 75:691-701.

Parada, E. \& S. Peredo. 2005. La relocalización como una herramienta de conservación y manejo de la biodiversidad. Lecciones aprendidas con Diplodon chilensis (Gray 1828) (Bivalvia, Hyriidae). Gayana 69 (1): 41-47.

Parada, E. \& S. Peredo. 2006. Estado de conocimiento de los bivalvos dulceacuícolas de Chile. Gayana 70 (1): 82-87.

Parada, E., S. Peredo, J. Valenzuela \& D. Manuschevich. 2007. Extention of the current Northern distribution range of freshwater mussel Diplodon chilensis (Gray, 1828) (Bivalvia: Hyriidae) in Chile. Gayana 71 (2): 212-215.

Prates, L. \& N. Marsans. 2007. El uso de moluscos de agua dulce (Diplodon chilensis patagonicus) en el sitio Angostura 1 (Departamento de General Conesa, Río Negro). Intersecciones en Antropología 8: 355-359.

Pavlovic, D., A. Troncoso, M. Massone \& R. Sánchez. 1998. La pequeña casa en la ladera; Blanca Gutiérrez (RML008), un asentamiento habitacional de la cultura Aconcagua. Boletín de la Sociedad Chilena de Arqueología 25: 13-18.

Quiroz, D. \& I. Martínez. 2007. Zooarqueología de la cueva de Quilmo, Chillán. En Actas del XVII Congreso Nacional de Arqueología Chilena, Valdivia. (En Prensa).

Rodríguez, A., R. Morales, C. González \& D. Jackson. 1993. Cerro La Cruz: Un enclave económico administrativo Incaico, curso medio del Aconcagua (Chile Central). Boletín del Museo Regional de La Araucanía 4: 201-234.

SÁnchez, M. \& J. InostrozA. 1985. Excavaciones arqueológicas en el Alero Quino1. Boletín del Museo Regional de La Araucanía (Temuco), 2:5362.

Sepúlveda, A. 1988. Estudio preliminar de la contaminación fecal en aguas naturales utilizando un organismo bioindicador y la técnica de filtración de membrana. Seminario para optar al título de Tecnólogo Médico. Universidad Austral de Chile. Valdivia. 50 pp.

Stehberg, R. \& A. Pinto. 1980. Ocupaciones Alfareras Tempranas quebrada El Salitral, Cordón de Chacabuco. Revista Chilena de Antropología 3: 57-73.

Troncoso, A., D. Pavlovich, C. Becker, P. González \& J. Rodríguez. 2004. Césped 3, Asentamiento del período Diaguita-Incaico sin cerámica Diaguita Fase III en el curso superior del río Illapel, IV Región, Chile. Chungará, Revista Chilena de Antropología, Volumen Especial; 893-906.

Valdés, C., M. SÁnchez, J. Inostroza, P. Sanzana \& X. NAVARro. 1982. Excavaciones arqueológicas en el Alero Quillen I, Provincia de Cautín, Chile. En Actas del IX Congreso Nacional de 
Antecedentes arqueológicos de Diplodon en Chile: JACKson, D. \& D. JACKson

Arqueología, pp. 399-435, Museo Arqueológico de La Serena.

Velásquez, H. \& L. Adán. 2002. Evidencias arqueofaunisticas del sitio Alero Marifilo1. Adaptación a los bosques templados de los sistemas lacustres del Centro-Sur de Chile. Boletín de la Sociedad Chilena de Arqueología 33-34:27-35.
Villagrán, C., P. Moreno \& R. Villa. 1995. Antecedentes palinológicos acerca de la historia cuaternaria de los bosques chilenos. En Ecología de los bosques nativos de Chile, Editado por J. Armesto, C. Villagrán \& M. Kalin, pp. 51-69. Editorial Universitaria, Santiago.

Recibido: 16.06 .08

Aceptado: 07.10 .08 